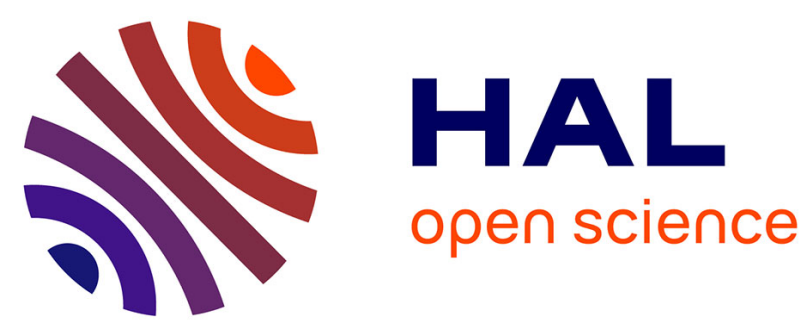

\title{
Probabilistic modeling of coupled heat transfer: A step towards optimization based on multiphysics Monte Carlo simulations
}

Christophe Spiesser, Victor Pozzobon, Olivier Farges, Jean-Jacques Bézian

\section{- To cite this version:}

Christophe Spiesser, Victor Pozzobon, Olivier Farges, Jean-Jacques Bézian. Probabilistic modeling of coupled heat transfer: A step towards optimization based on multiphysics Monte Carlo simulations. International Journal of Thermal Sciences, 2018, 132, pp.387-397. 10.1016/j.ijthermalsci.2018.04.004 . hal-01822505

\section{HAL Id: hal-01822505 https://hal.science/hal-01822505}

Submitted on 29 Jun 2018

HAL is a multi-disciplinary open access archive for the deposit and dissemination of scientific research documents, whether they are published or not. The documents may come from teaching and research institutions in France or abroad, or from public or private research centers.
L'archive ouverte pluridisciplinaire HAL, est destinée au dépôt et à la diffusion de documents scientifiques de niveau recherche, publiés ou non, émanant des établissements d'enseignement et de recherche français ou étrangers, des laboratoires publics ou privés. 


\title{
Probabilistic modeling of coupled heat transfer: A step towards optimization based on multiphysics Monte Carlo simulations
}

\author{
C. Spiesser ${ }^{\mathrm{a}, *}$, V. Pozzobon ${ }^{\mathrm{b}}$, O. Farges ${ }^{\mathrm{a}}$, J.J. Bézian ${ }^{\mathrm{a}}$ \\ a École des Mines d'Albi-Carmaux, Campus Jarlard, UMR CNRS 5302 - Centre de Recherche d'Albi en génie des Procédés des Solides Divisés, de l'Énergie et de \\ l'Environnement (RAPSODEE), F-81013 Albi CT cedex 09, France \\ b LGPM, CentraleSupélec, Université Paris-Saclay, SFR Condorcet FR CNRS 3417, Centre Européen de Biotechnologie et de Bioéconomie (CEBB), chemin des Sohettes, \\ 51110 Pomacle, France
}

A R T I C L E I N F O

\section{Keywords:}

Heat transfer

Coupling

Self-adaptive

Multiphysics

Monte Carlo method

\begin{abstract}
A B S T R A C T
This article presents a modular framework allowing to construct probabilistic models of coupled heat transfer problems in complex systems. First, a substructuring approach has been applied to formalize the problem. This process allowed for the coupling of physical field submodels, in our case temperature and radiative intensity. Each physical model was established according to the conservation law inside of its domain (solid and fluid) and the continuity laws at interfaces. Then, these models have been rewritten from the deterministic point of view to a probabilistic one. This enables a recursive Monte Carlo algorithm to estimate the desired values. After a validation stage, against academic cases, this framework is applied to examples emulating heat transfer in buildings. This approach presents a major beneficial behavior for complex systems optimization: only the influential parts of the problem have an effect on the computational time. These regions are automatically identified in a self-adaptive way, even in intricate or extensive geometries.
\end{abstract}

\section{Introduction}

For decades, the optimization processes have enabled engineers and designers to improve systems during their design stages. Taking advantage of the increasing computational power, they now wish to optimize more and more complex systems (complicated geometry, multivariate and/or multi-objective design, multi-scale phenomena, multiphysics problems). Simulation-based optimization methods are aiming at finding, by an iterative process, an extremum of a function, known as the objective function, which is evaluated thanks to the simulation results [1-3]. To perform these simulations, the most spread methods are the grid-based quadratures. Yet their use usually requires to describe complex systems by a great number of mesh elements owing to tortuosity, extensive geometry and/or multi-scale problems. Furthermore a vast grid implies a proportional need of computing power and memory to run a simulation. This limitation becomes all the more important when the complexity of systems is increasing.

Another kind of quadrature exists: the stochastic grid-free quadratures among which the Monte Carlo method is found. This method was first formalized by Metropolis and Ulam, in 1949, in the neutron diffusion field of science [4]. Fifteen years later, Hammersley and Handscomb highlighted the opportunity to use this method in a large variety of other domains among which the solution of linear operator equations, statistical mechanics or polymer science [5]. This approach allows for a stochastic estimation of a quantity of interest. The estimation is the mean value and the standard error of a great number of independent evaluations of a probabilistic model. Its two main characteristics are being a meshless quadrature and presenting a convergence rate independent of the problem dimension. Consequently, the higher the number of problem dimensions and the more competitive the Monte Carlo method is, compared to grid-based quadratures. Indeed, the laters follow a decreasing convergence rate when the problem dimension increase [6,7]. That is why even today the Monte Carlo method is mostly restricted to problems described by more than 3 dimensions such as radiative transfer, finance, particle physics, acoustics, cosmological models $[8,9]$. The drawback of a meshless approach is that the result is a single value (and its standard error), which can be a function evaluated locally or integrated over a domain. This could be a considerable disadvantage if you are looking to the whole unknown field. But, when using an optimization process, only the objective function needs to be assessed, not the whole field. Hence, that main drawback of the Monte Carlo grid-free approach can easily be overcome for an optimization process. Indeed, using the Monte Carlo method to estimate directly the objective function will only cost a single

\footnotetext{
* Corresponding author.

E-mail address: christophe.spiesser@mines-albi.fr (C. Spiesser).
} 


\section{Nomenclature}

$c_{p \Omega} \quad$ Specific heat capacity of the $\Omega$ domain $\left[J . \mathrm{kg}^{-1} \cdot \mathrm{K}^{-1}\right]$

$\boldsymbol{d}_{i, j} \quad$ Direction vector defined at the $\boldsymbol{r}_{i, j}$ point [m]

$\partial \quad$ Thermal dissipation coefficient $\left[W \cdot \mathrm{m}^{-2} \cdot \mathrm{K}^{-1}\right]$

$\mathbf{H}^{\Omega+}(\boldsymbol{x})$ Hemisphere of the directions incident from the $\Omega$ domain at the $\boldsymbol{x}$ point (identifying name)

$\mathbf{H}^{\Omega-}(\boldsymbol{x})$ Hemisphere of the directions leaving the $\boldsymbol{x}$ point towards the $\Omega$ domain (identifying name)

$h_{\partial \Omega}^{\Omega} \quad$ Convection coefficient at the interface $\partial \Omega$, on the $\Omega$ fluid domain side $\left[W . m^{-2} \cdot K^{-1}\right]$

$I(\boldsymbol{x}, \omega)$ Total directional intensity field at the $\boldsymbol{x}$ point towards the $\omega$ direction $\left[W . m_{\perp}^{-2} . s r^{-1}\right]$

$\langle i+j\rangle \quad$ Mean length of the exploration paths used to compute the estimation (calculated in number of interaction points)

$\dot{m}_{\Omega+} \quad$ Total mass flow rate leaving the $\Omega$ fluid domain (positively defined) $\left[\mathrm{kg} . \mathrm{s}^{-1}\right]$

$\dot{m}_{\Omega-}\left(\Omega_{e}\right)$ Mass flow rate coming from the $\Omega_{e}$ fluid domain into the $\Omega$ domain (positively defined) $\left[\mathrm{kg} . \mathrm{s}^{-1}\right]$

$\boldsymbol{n}_{\partial \Omega}^{\Omega+} \quad$ Normal direction vector at the boundary $\partial \Omega$ leaving the $\Omega$ domain $[m]$

$\boldsymbol{n}_{\text {cond }} \quad$ Direction vector along which the conductive heat transfer takes place (1D plane model) $[\mathrm{m}]$

$P$ (event) Probability that the event occurs

$p_{R V} \quad$ Probability density function associated with the $R V$ random variable

$\dot{q}^{\Omega+} \quad$ Total net flux density leaving the $\Omega$ domain $\left[W . \mathrm{m}^{-2}\right]$

$\dot{Q}_{\text {mode }}^{\Omega+} \quad$ Net flux leaving the $\Omega$ domain by the mode of heat transfer (conduction, convection, radiation or enthalpy) $[W]$

$\dot{q}_{\text {mode }}^{\Omega+} \quad$ Net flux density leaving the $\Omega$ domain by the mode of heat transfer (conduction, convection, radiation or enthalpy) [W. $\left.\mathrm{m}^{-2}\right]$ $\dot{q}_{s r c}^{2 D} \quad$ Flux density of surfacic heat source at an interface $\left[W . m^{-2}\right]$

$\boldsymbol{r}_{i, j} \quad$ Vector of the j-th position of the total directional intensity exploration path/series starting at the $\boldsymbol{x}_{i}$ point $[\mathrm{m}]$

$s_{\partial \Omega}^{\Omega} \quad$ Specularity ratio for the reflection at the interface $\partial \Omega$, on the $\Omega$ domain side

$T(\boldsymbol{x}) \quad$ Temperature field at the $\boldsymbol{x}$ point $[K]$

$T_{\text {ref }} \quad$ Reference temperature used for the radiative transfer linearization $[K]$

$\hat{w}_{\text {event }} \quad$ Monte Carlo weight associated to the event occurrence

$\boldsymbol{X}_{i} \quad$ Random variable of the i-th position vector $\boldsymbol{x}_{i}[\mathrm{~m}]$

$\boldsymbol{x}_{i} \quad$ Vector of the i-th position of the exploration path/series $[m]$

$\boldsymbol{x}_{i}^{\in \Omega} \quad:=\boldsymbol{x}_{i} \in \Omega[m]$

$\boldsymbol{x}_{i}^{\in \partial \Omega} \quad:=\boldsymbol{x}_{i} \in \partial \Omega[\mathrm{m}]$

Greek symbols

$\alpha_{\partial \Omega}^{\Omega} \quad$ Total hemispherical absorpivity of $\partial \Omega$ boundary, on the $\Omega$ domain side

$\varepsilon_{\partial \Omega}^{\Omega} \quad$ Total hemispherical emissivity of $\partial \Omega$ boundary, on the $\Omega$ domain side

$\lambda_{\Omega} \quad$ Thermal conductivity of the $\Omega$ domain $\left[W \cdot \mathrm{m}^{-1} \cdot \mathrm{K}^{-1}\right]$

$\Omega \quad$ Volumetric domain (identifying name)

$\partial \Omega \quad$ Domain boundary (identifying name)

$\rho_{\partial \Omega}^{\Omega} \quad$ Total hemispherical reflectivity at the interface $\partial \Omega$, on the $\Omega$ domain side

$\begin{array}{ll}\sigma & \text { Stefan-Boltzmann constant: } 5.67 \times 10^{-8}\left[\mathrm{~W} \cdot \mathrm{m}^{-2} \cdot \mathrm{K}^{-4}\right] \\ \sigma_{t} & \text { Standard error of the computational time estimation }(\mathrm{t})[\mathrm{s}]\end{array}$

$\sigma_{T} \quad$ Standard error of the temperature estimation (T) $[K]$

$\tau_{\partial \Omega} \quad$ Total hemispherical transmittivity for the interface $\partial \Omega$ estimation.

According to the Los Alamos National Laboratory [10], two of the main limitations to the Monte Carlo method spreading in simulationbased optimization are: the ability to evaluate nonlinear functions of integrals and the coupling issue because complex systems are generally multiphysic. Recent works [11-13] are bringing perspectives in order to alleviate the first challenge. We will focus on the second one, which is also a current concern for the grid-based methods and is shared by a wide variety of fields from astrophysics to biochemistry passing by nuclear reactor engineering or rarefied gas [14-18]. When dealing with multiphysic problems, the most spread approach consists in trying to couple several models or even simulation codes, one for each physical phenomenon, in a iterative converging process. The main difficulty is to keep coherent results from different tools, which rely on their own assumptions and have sometimes radically different solving strategies.

Regarding more specifically coupled heat transfer, and by instance the coupling of conduction and radiative transfer, we can find in the litterature different examples. Originally motivated by reducing computational time within a context of lesser available computational power, numerous attempts to couple grid-based methods for both radiative and conductive heat transfer can be found [19-26]. That approach has the advantage of coupling two similar methods but suffers twice of the drawbacks of grid-based quadratures as the systems become more and more complex. Furthermore, examples of coupling a grid-free method with a finite volumes or elements method can be found in literature [27-31]. Usually, in these cases, the Monte Carlo method is used to model the radiative transfer. These attempts have spread thanks to the increasing availability of computational power but are especially difficult to implement owing to the heterogeneity of the techniques which have to cooperate together. Finally, the third possibility is to use the Monte Carlo method to solve the whole problem. That approach allows to take advantage from both model characteristics homogeneity and the aforementioned attractive features of the Monte Carlo method. This goal was announced by the presentation of a first draft, in collaboration with the Meso-Star start-up, during the CTRPM-V conference [32]. Nevertheless, according to the best of authors' knowledge, this way of coupling has not been investigated yet. Although, Vignoles proposed very recently a method dedicated to simulation inside of porous media by coupling two Monte Carlo Random Walks, one for each phenomenon, in Ref. [33]. The idea is to follow the spreading of a "walker" population into the porous medium by both a radiative random walk and a conductive one. Each "walker" carrying a "quantum of excess enthalpy". At the end of a simulation, the temperature perturbation field is approximated by counting the number of "walker" in each discretized volume element. Even if this technique is also named "Monte Carlo", it features important differences with the Monte Carlo method used in the present article which is using neither a volume discretization nor following an energy quantum carrier population.

In order to solve the whole problem, i.e. coupled condution, convection and radiative heat transfer, using a single Monte Carlo algorithm, the prerequisite is to get a probabilistic model of the system. However, the more complex the system, the more difficult its modeling step. Therefore, to face this challenge, we have created a framework easing the construction stages, by using a systemic substructuring approach. This tool allows to assemble components, which have been already independently modeled. This approach provides several benefits: modularity, versatility and ease to upgrade. The aim of this article is to show how such an approach can be applied to fully coupled heat transfer problems. First we describe the substructuring approach and how the Monte Carlo algorithm can be used to solve modular models. Next, each submodel is detailed. Then the reliability of this approach is assessed by validating the framework results against academic cases. Finally, an application to heat transfer in a building will show the practical advantages of this strategy. 


\section{A recursive Monte Carlo algorithm to solve the modular models}

The proposed framework is built on a systemic approach, which models a complex system as a group of elementary components potentially interacting with each other. Applied to grid-based deterministic quadratures, this kind of modeling process is known as the Model Synthesis approach. This has been firstly introduced in 1993 by Ebert and Nataf $[34,35]$ and then developed by Lefebvre [36-38].

In the following, systems are modeled by assembling three kinds of 3D components: solid domains, fluid domains and interfaces. A set of assembled components is called a scene. In each component, the physical fields are described by either a model depending on its surrounding or by an explicit condition. The expression accounting for the model surrounding could be the field value at other points or in other components for instance. To apply this approach to coupled heat transfer, we have used two physical fields: the temperature and the total directional intensity for radiative heat transfer. The models of these physical fields in the different kinds of components will be made explicit in the next section. But before, we have to understand how the Monte Carlo algorithm will solve the problem modeled as a scene. To explain that process, we will use an example (Fig. 1).

As aforementioned, a Monte Carlo algorithm is a stochastic estimation process. Values are determined by averaging the results of a large number of evaluations of the probabilistic function. Each evaluation is named an history. In the coupled heat transfer case, one of the simplest objective functions could be a temperature value at a specific point in the scene. As an example, we are going to evaluate this objective function at a point $x_{i}$. We have previously explained that the temperature at $x_{i}$ can be known in two forms: as an explicit condition or as a model. If it is an explicit condition, the data is directly obtained and the evaluation is done. If it is known as a model, the algorithm has to evaluate it. Models are generally a sum of several contributions, each having an associated probability. Consequently, to evaluate the model, firstly a stochastic process is used to choose a contribution, proportionally to its probability, then the chosen contribution is evaluated. The simplest contribution possible is the value of the physical field, here the temperature, at an other point, which may originate from another component. So if the temperature model at $x_{i}$ depends on the temperature at two other points $y_{1}$ and $y_{2}$, the stochastic process will randomly choose one of them: the $y_{2}$ contribution for example. So to evaluate the temperature at $x_{i}$, the algorithm needs now to evaluate the temperature at $x_{i-1}:=y_{2}$, which can also be known in one of the two forms. This will create an exploration path of the scene by generating a series of positions with this recursive stochastic process. This path ends when an explicit data is found, which is generally a boundary value of the problem. Thanks to this explicit data, the model value at the upstream point can be evaluated, and so on until the algorithm reaches the first point $x_{i}$ of the series in an inverse cascade process. By using a stochastic process to pick a single direction/contribution to extend the exploration path, a well convergent behavior is obtained for this recursive evaluation algorithm.

\section{Probabilistic modeling of physical fields in components}

The following models are based on two main physical laws: the energy conservation inside domains (regardless their kind) and the continuity principle of physical fields and their associated flux densities at interfaces. On top of that, four general assumptions have been made: the conduction, convection and radiative transfer are in steady state; they can be described by linear models; domains can be either opaque or totally transparent; and finally parameters and physical properties are constant. Hence, the temperature field inside domains is governed by: conduction for solid domains and convection for fluid domains. Furthermore, interfaces can potentially exchange energy by conduction with the surrounding solids, by convection with the fluid domains next to them and/or by radiative heat transfer with other interfaces through a transparent domain.

\subsection{Temperature field inside solid domains}

Deterministic model. Let $\Omega$ be a solid and homogeneous domain with no heat source where a 1D conductive heat transfer takes place along the direction $\boldsymbol{n}_{\text {cond }}$ between two planes. This domain is defined by two parallel planar boundaries ( $\partial \Omega_{-}$and ) where the temperature is known (explicitly or as a model). By applying the energy conservation law in a such domain, a well known results is obtained, the temperature field has a linear expression linking the temperature value at each boundary: $T\left(\boldsymbol{x}_{i}^{\in \partial \Omega_{-}}\right)$and $T\left(\boldsymbol{x}_{i}^{\in \partial \Omega_{+}}\right)$(Eq. (1) and Fig. 2). That result will serve as temperature field model in solid domains.

$T\left(\boldsymbol{x}_{i}^{\in \Omega}\right)=\left\{\begin{array}{c}T\left(\boldsymbol{y}_{-n}^{\in \partial \Omega_{-}}\right) \\ +\frac{\left\|\boldsymbol{x}_{i}^{\in \Omega}-\boldsymbol{y}_{-n}^{\in \partial \Omega_{-}}\right\|}{\| \boldsymbol{y}_{+n}^{\in \partial \Omega_{+}}-\boldsymbol{y}_{-n}^{\in \partial \Omega_{-} \|}}\left(T\left(\boldsymbol{y}_{+n}^{\in \partial \Omega_{+}}\right)-T\left(\boldsymbol{y}_{-n}^{\in \partial \Omega_{-}}\right)\right)\end{array}\right.$

Probabilistic model. Eq. (1) is a deterministic model of the temperature field in solid domains, nevertheless in order to evaluate it by the stochastic Monte Carlo method it has to be turned into a probabilistic equation. Two different contributions can be identified in that model: the temperature field influence from both boundaries. By rearranging this model, some barycentric weightings can appear before each temperature field evaluation. They can be used as probabilities to extend the exploration path towards a boundary rather than the other. This probability will be written as the probability of a point $\boldsymbol{y}^{\in \partial \Omega}$ at the boundary $\partial \Omega$ to be the next position $\boldsymbol{x}_{i-1}$ in the exploration path i.e. $P\left(\boldsymbol{x}_{i-1}:=\boldsymbol{y}^{\in \partial \Omega}\right)$. The associated remaining part of the contribution, which will be explicitly evaluated, is named a weight and is written $\hat{w}\left(\boldsymbol{x}_{i-1}\right)$. Finally, the deterministic equation (Eq. (1)) is equivalent to the following probabilistic model (Eq. (2)):

$T\left(\boldsymbol{x}_{i}^{\in \Omega}\right)=\left\{\begin{array}{c}P\left(\boldsymbol{x}_{i-1}:=\boldsymbol{y}_{-n}^{\in \partial \Omega_{-}}\right) \times \hat{w}_{\partial \Omega_{-}}\left(\boldsymbol{x}_{i-1}^{\in \partial \Omega_{-}}\right) \\ +\quad P\left(\boldsymbol{x}_{i-1}:=\boldsymbol{y}_{+n}^{\in \partial \Omega_{+}}\right) \times \hat{w}_{\partial \Omega_{+}}\left(\boldsymbol{x}_{i-1}^{\in \partial \Omega_{+}}\right)\end{array}\right.$

Where

$\left\{\begin{array}{l}\exists e_{-} \in \mathbb{R}^{+*} \mid \boldsymbol{y}_{-n}=\boldsymbol{x}_{i}^{\in \Omega}-e_{-} \times \boldsymbol{n}_{\text {cond }} \& \boldsymbol{y}_{-n} \in \partial \Omega_{-} \\ P\left(\boldsymbol{x}_{i-1}:=\boldsymbol{y}_{-n}^{\in \partial \Omega_{-}}\right)=\frac{\left\|\boldsymbol{y}_{+n}^{\in \partial \Omega_{+}}-\boldsymbol{x}_{i}^{\in \Omega_{\|}}\right\|}{\| \boldsymbol{y}_{+n}^{\in 2 \Omega_{+}}-\boldsymbol{y}_{-n}^{\in \partial \Omega_{-} \|}} \\ \hat{w}_{\partial \Omega_{-}}\left(\boldsymbol{x}_{i-1}^{\in \partial \Omega_{-}}\right)=T\left(\boldsymbol{x}_{i-1}^{\in \partial \Omega_{-}}\right)\end{array}\right.$
$\left\{\begin{array}{l}\exists e_{+} \in \mathbb{R}^{+*} \mid \boldsymbol{y}_{+n}=\boldsymbol{x}_{i}^{\in \Omega}+e_{+} \times \boldsymbol{n}_{\text {cond }} \& \boldsymbol{y}_{+n} \in \partial \Omega_{+} \\ P\left(\boldsymbol{x}_{i-1}:=\boldsymbol{y}_{+n}^{\in \partial \Omega_{+}}\right)=\frac{\| \boldsymbol{x}_{i}^{\in \Omega}-\boldsymbol{y}_{-n}^{\in \partial \Omega_{-} \|}}{\| \boldsymbol{y}_{+n}^{\in \partial \Omega_{+}}-\boldsymbol{y}_{-n}^{\in \partial \Omega_{-} \|}} \\ \hat{w}_{\partial \Omega_{+}}\left(\boldsymbol{x}_{i-1}^{\in \partial \Omega_{+}}\right)=T\left(\boldsymbol{x}_{i-1}^{\in \partial \Omega_{+}}\right)\end{array}\right.$

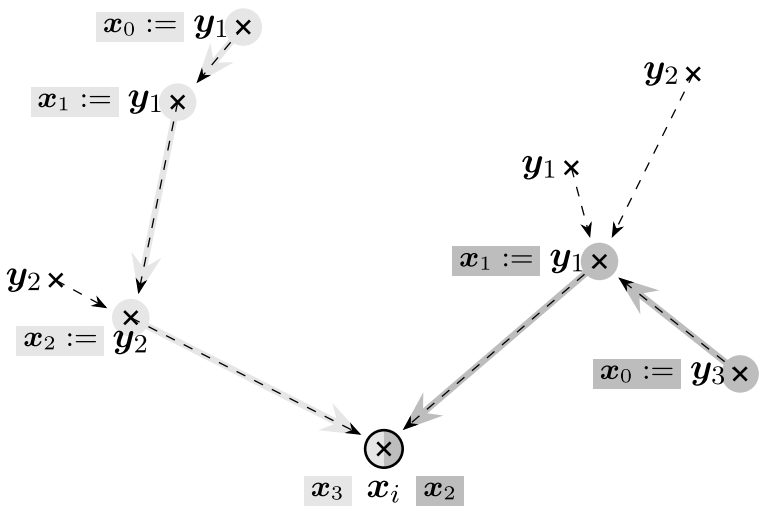

probe $\quad-\rightarrow$ contribution history $1 \quad$ history 2

Fig. 1. Diagram representing exploration paths construction to evaluate a field value at $x_{i}$ 


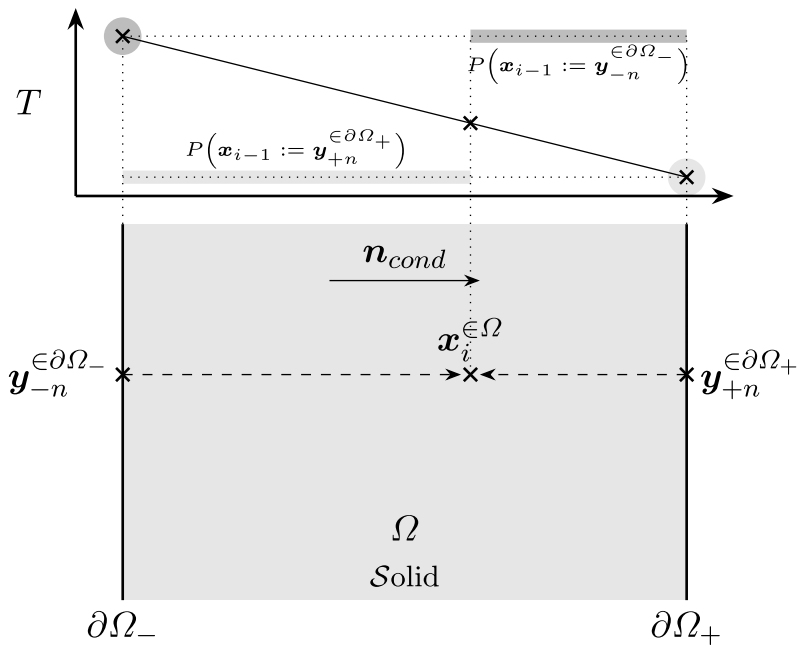

Fig. 2. Diagram representing the notations used in the temperature model for points inside solid domains.

\subsection{Temperature field inside fluid domains}

Deterministic model. To model the temperature field inside fluid domains, without having to take into account fluid dynamics, specific assumptions have been made. Firstly, fluid domains are considered as perfectly mixed hence their temperature field is uniform in each domain. On top of that, the convection is seen as a macroscopical phenomenon and is modeled by a convection coefficient, $h_{\partial \Omega}^{\Omega}\left(\boldsymbol{y}_{\text {conv }}^{\in \partial \Omega}\right)$.

In the case of fluid domains, we consider two kinds of energy fluxes: the convection between the fluid and its boundaries and enthalpy fluxes which are a consequence of mass exchanges between several fluid domains.

On one hand, the convective flux leaving the $\Omega$ domain is obtained by integrating the convective flux density over the domain boundary $\partial \Omega$ (Fig. 3 and Eq. (3)).

$\dot{Q}_{\text {conv }}^{\Omega+}\left(\boldsymbol{x}_{i}^{\in \Omega}\right)=\int_{\partial \Omega} h_{\partial \Omega}^{\Omega}\left(\boldsymbol{y}_{\text {conv }}^{\in \partial \Omega}\right) \times\left(T\left(\boldsymbol{x}_{i}^{\in \Omega}\right)-T\left(\boldsymbol{y}_{\text {conv }}^{\in \partial \Omega}\right)\right) \mathrm{d} \boldsymbol{y}_{\text {conv }}$

On the other hand, the net enthalpy flux leaving the $\Omega$ fluid domain is the difference between the total leaving enthalpy flux and the sum of fluxes coming from the $n$ other fluid domain(s) $\Omega_{e}$ (Eq. (4)).

$\dot{Q}_{\text {enth }}^{\Omega+}\left(\boldsymbol{x}_{i}^{\in \Omega}\right)=\left\{\begin{array}{c}\dot{m}_{\Omega+} c_{p \Omega} \times T\left(\boldsymbol{x}_{i}^{\in \Omega}\right) \\ -\sum_{e=1}^{n} \dot{m}_{\Omega-}\left(\Omega_{e}\right) c_{p \Omega} \times T\left(\boldsymbol{y}_{\text {enth }, e}^{\in \Omega_{e}}\right)\end{array}\right.$

Where $c_{p \Omega}$ is the specific heat capacity of $\Omega, \dot{m}_{\Omega-}\left(\Omega_{e}\right)$ is the mass flow rate coming from the $\Omega_{e}$ domain into $\Omega$ (positively defined), and $\dot{m}_{\Omega+}$ is the total mass flow rate leaving $\Omega$ (positively defined). The mass and energy conservation laws establish that, in steady state, there are neither mass (Eq. (5)) nor energy (Eq. (6)) accumulation in the domain.

$\dot{m}_{\Omega+}=\sum_{e=1}^{n} \dot{m}_{\Omega-}\left(\Omega_{e}\right)$

$$
\begin{aligned}
0= & \int_{\partial \Omega} h_{\partial \Omega}^{\Omega}\left(\boldsymbol{y}_{\text {conv }}\right) \times\left(T\left(\boldsymbol{x}_{i}^{\in \Omega}\right)-T\left(\boldsymbol{y}_{\text {conv }}\right)\right) \mathrm{d} y_{\text {conv }}+\dot{m}_{\Omega+} c_{p \Omega} \times T\left(\boldsymbol{x}_{i}^{\in \Omega}\right) \\
& -\sum_{e=1}^{n} \dot{m}_{\Omega-}\left(\Omega_{e}\right) c_{p \Omega} \times T\left(\boldsymbol{y}_{\text {enthe }, e}^{\in \Omega_{e}}\right)
\end{aligned}
$$

Eqs. (5) and (6) can be rearranged in the following deterministic model of the temperature in fluid domains (Eq. (7)):
$T\left(\boldsymbol{x}_{i}^{\in \Omega}\right)=\left\{\begin{array}{l}\frac{\int_{\partial \Omega} h_{\partial \Omega}^{\Omega}(\boldsymbol{y}) \mathrm{d} \boldsymbol{y}}{\dot{m}_{\Omega+} c_{p \Omega}+\int_{\partial \Omega} h_{\partial \Omega}^{\Omega}(\boldsymbol{y}) \mathrm{d} \boldsymbol{y}} \times \int_{\partial \Omega} \frac{h_{\partial \Omega}^{\Omega}\left(\boldsymbol{y}_{\text {conv }}\right)}{\int_{\partial \Omega} h_{\partial \Omega}^{\Omega}(\boldsymbol{y}) \mathrm{d} \boldsymbol{y}} \times T\left(\boldsymbol{y}_{\text {conv }}\right) \mathrm{d} \boldsymbol{y}_{\text {conv }} \\ +\sum_{e=1}^{n} \frac{\dot{m}_{\Omega-}\left(\Omega_{e}\right) c_{p \Omega}}{\dot{m}_{\Omega+} c_{p \Omega}+\int_{\partial \Omega} h_{\partial \Omega}^{\Omega}(\boldsymbol{y}) \mathrm{d} \boldsymbol{y}} \times T\left(\boldsymbol{y}_{\text {enth }, e}^{\in \Omega_{e}}\right)\end{array}\right.$

Probabilistic model. We can see on the first line of Eq. (7) the convection contribution coming from the boundary and below the sum of the all coming enthalpy contributions from $\Omega_{e}$ domains. It can be rewritten in a probabilistic manner by adding probabilities and associated weights. A probability density function, written $p_{\boldsymbol{X}_{i-1}}\left(\boldsymbol{x}_{i-1}\right)$, is therefore introduced. It will be used to sample a point over the $\partial \Omega$ boundary, in order to evaluate the integral by the Monte Carlo method. Thus, the following probabilistic model is obtained (Eq. (8)):

$T\left(\boldsymbol{x}_{i}^{\in \Omega}\right)=\left\{\begin{array}{c}P\left(\boldsymbol{x}_{i-1}:=\boldsymbol{y}_{\text {conv }}^{\in \partial \Omega}\right) \times \int_{\partial \Omega} p_{\boldsymbol{X}_{i-1}}\left(\boldsymbol{x}_{i-1}\right) \mathrm{d} \boldsymbol{x}_{i-1} \times \hat{w}_{\text {conv }}\left(\boldsymbol{x}_{i-1}^{\in \partial \Omega}\right) \\ +\sum_{e=1}^{n} P\left(\boldsymbol{x}_{i-1}:=\boldsymbol{y}_{\text {enth }, e}^{\in \Omega_{e}}\right) \times \hat{w}_{\text {enth }, e}\left(\boldsymbol{x}_{i-1}^{\in \Omega_{e}}\right)\end{array}\right.$

Where

$\left\{\begin{array}{l}\exists \boldsymbol{y}_{\text {conv }} \in \partial \Omega \\ P\left(\boldsymbol{x}_{i-1}:=\boldsymbol{y}_{\text {conv }}^{\in \partial \Omega}\right)=\frac{\int_{\partial \Omega} h_{\partial \Omega}^{\Omega}(\boldsymbol{y}) \mathrm{d} \boldsymbol{y}}{\dot{m}_{\Omega+}+c_{p \Omega}+\int_{\partial \Omega} h_{\partial \Omega}^{\Omega}(\boldsymbol{y}) \mathrm{d} \boldsymbol{y}} \\ p_{\boldsymbol{X}_{i-1}}\left(\boldsymbol{x}_{i-1}\right)=\frac{h_{\partial \Omega}^{\Omega}\left(\boldsymbol{x}_{i-1}\right)}{\partial_{\partial \Omega} h_{\partial \Omega}^{\Omega}(\boldsymbol{y}) \mathrm{d} \boldsymbol{y} \boldsymbol{y}} \\ \hat{w}_{\text {conv }}\left(\boldsymbol{x}_{i-1}^{\in \partial \Omega}\right)=T\left(\boldsymbol{x}_{i-1}^{\in \partial \Omega}\right)\end{array}\right.$
$\left\{\begin{array}{l}\exists \boldsymbol{y}_{\text {enth }, e} \in \Omega_{e} \\ P\left(\boldsymbol{x}_{i-1}:=\boldsymbol{y}_{\text {enthee }}^{\in \Omega_{e}}\right)=\frac{\dot{m}_{\Omega-}\left(\Omega_{e}\right) c_{p \Omega}}{\dot{m}_{\Omega+} c_{p \Omega}+\int_{\partial \Omega} h_{\partial \Omega}^{\Omega}(\boldsymbol{y}) \mathrm{d} \boldsymbol{y}} \\ \hat{w}_{\text {enth,e }}\left(\boldsymbol{x}_{i-1}^{\in \Omega_{e}}\right)=T\left(\boldsymbol{x}_{i-1}^{\in \Omega_{e}}\right)\end{array}\right.$

\subsection{Temperature field at an interface}

Deterministic model. An interface can eventually delimit two different domains, generically named $\Omega_{1}$ and $\Omega_{2}$. In this case, the interface is a shared boundary ( $\mathrm{I}=\partial \Omega_{1} \bigcap \partial \Omega_{2}$ ). The interface $\mathcal{I}$ can also delimit a single domain and in this case, the interface is a boundary of the scene. In order to remain faithful to our chosen generalized and systemic approach, we have developed a single model for both cases.

As aforementioned, we have used the continuity law of the physical field (here the temperature) and the flux density associated between the both sides of each interface to build the following model (Eq. (9) and Figs. 4-6).

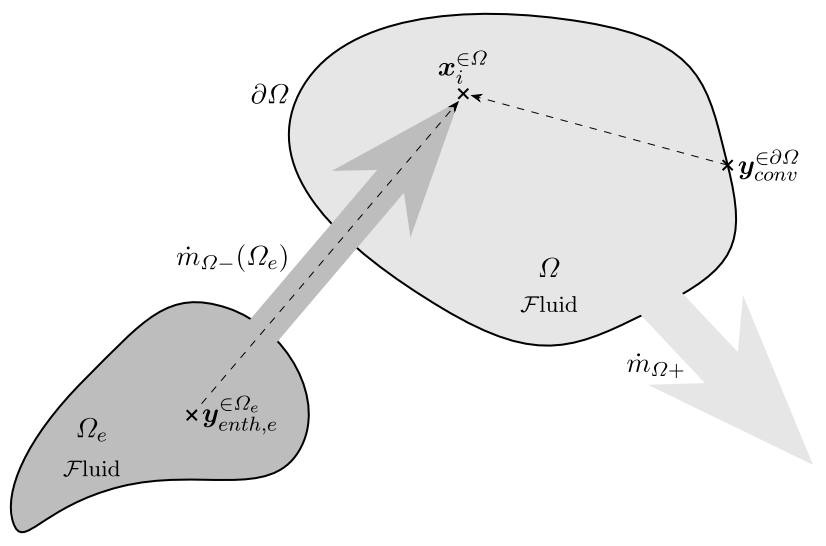

Fig. 3. Diagram representing the notations used in the temperature model for points inside fluid domains. 


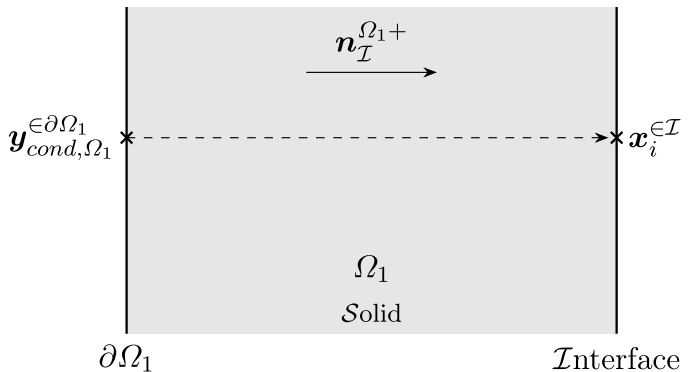

Fig. 4. Diagram representing the notations used in the temperature model for points on an interface under conductive heat flux.

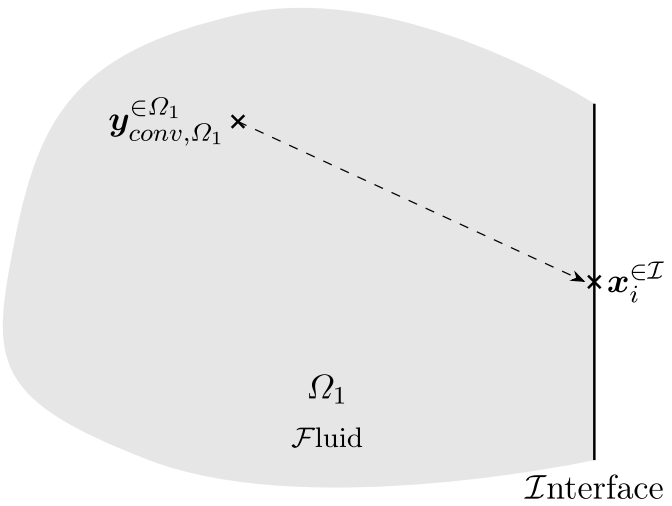

Fig. 5. Diagram representing the notations used in the temperature model for points on an interface under convective heat flux.

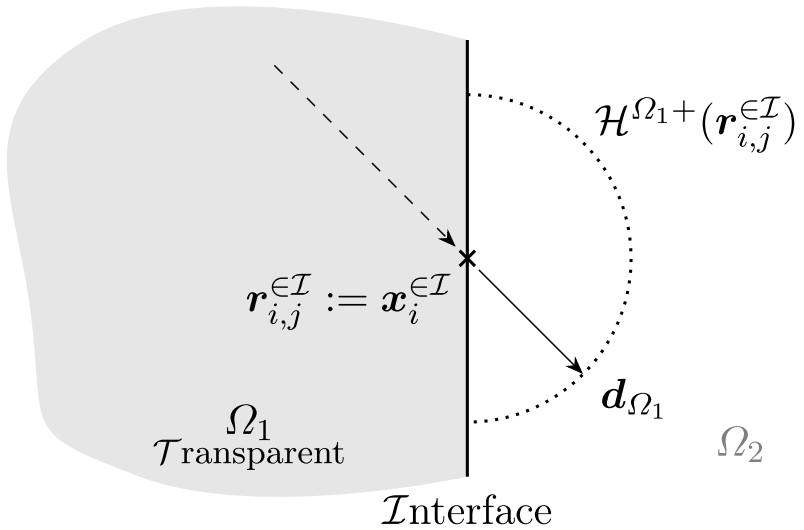

Fig. 6. Diagram representing the notations used in the temperature model for points on an interface under radiative heat flux.

$$
\begin{aligned}
0= & \dot{q}^{\Omega_{1}+}\left(\boldsymbol{x}_{i}^{\in I}\right)+\dot{q}^{\Omega_{2}+}\left(\boldsymbol{x}_{i}^{\in I}\right)+\dot{q}_{s r c}^{2 D}\left(\boldsymbol{x}_{i}^{\in I}\right) \\
0= & \dot{q}_{c o n d}^{\Omega_{1}+}\left(\boldsymbol{x}_{i}^{\in I}\right)+\dot{q}_{c o n v}^{\Omega_{1}+}\left(\boldsymbol{x}_{i}^{\in I}\right)+\dot{q}_{r a d}^{\Omega_{1}+}\left(\boldsymbol{x}_{i}^{\in I}\right) \\
& +\dot{q}_{c o n d}^{\Omega_{2}+}\left(\boldsymbol{x}_{i}^{\in I}\right)+\dot{q}_{c o n v}^{\Omega_{2}+}\left(\boldsymbol{x}_{i}^{\in I}\right)+\dot{q}_{r a d}^{\Omega_{2}+}\left(\boldsymbol{x}_{i}^{\in I}\right) \\
& +\dot{q}_{s r c}^{2 D}\left(\boldsymbol{x}_{i}^{\in I}\right)
\end{aligned}
$$

Complementary assumptions have been made for radiative transfer: the interfaces are modeled as gray surfaces under the Local Thermodynamic Equilibrium assumption. On top of that, a linearized model for the radiative transfer has been built using a Taylor expansion at the vicinity of an arbitrary reference temperature, $T_{r e f}$. This implies a $100 \mathrm{~K}$ maximum contrast among the scene elements. Furthermore, it is possible to develop each flux density expression (Eq. (10)).

$$
\begin{aligned}
& 0=-\lambda_{\Omega_{1}} \frac{T\left(x_{i} \in I\right)-T\left(y_{\text {cood }}^{\in \partial \Omega_{1}}, \Omega_{1}\right)}{\left\|\boldsymbol{x}_{i} \in I-\boldsymbol{y}_{\text {cond }, \Omega_{1} \|}\right\|} \\
& +h_{I}^{\Omega_{1}}\left(\boldsymbol{x}_{i}^{\in I}\right) \times\left(T\left(\boldsymbol{y}_{\text {conv }, \Omega_{1}}^{\in \Omega_{1}}\right)-T\left(\boldsymbol{x}_{i}^{\in I}\right)\right) \\
& +\varepsilon_{I}^{\Omega_{1}}\left(\boldsymbol{x}_{i}^{\in I}\right) \times\left\{\begin{array}{l}
\int_{\mathcal{H}^{\Omega_{1}+}\left(\boldsymbol{x}_{i} \in I\right)}\left(\boldsymbol{d}_{\Omega_{1}} \bullet \boldsymbol{n}_{I}^{\Omega_{1}+}\left(\boldsymbol{x}_{i}^{\in I}\right)\right) I\left(\boldsymbol{x}_{i}^{\in I}, \boldsymbol{d}_{\Omega_{1}}\right) \mathrm{d} \boldsymbol{d}_{\Omega_{1}} \\
-\sigma \times\left(4 T_{\text {ref }}^{3} T\left(\boldsymbol{x}_{i}^{\in I}\right)-3 T_{\text {ref }}^{4}\right)
\end{array}\right.
\end{aligned}
$$

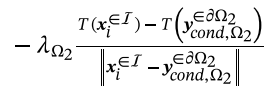

$$
\begin{aligned}
& +h_{I}^{\Omega_{2}}\left(\boldsymbol{x}_{i}^{\in I}\right) \times\left(T\left(\boldsymbol{y}_{\text {conv }, \Omega_{2}}^{\in \Omega_{2}}\right)-T\left(\boldsymbol{x}_{i}^{\in I}\right)\right) \\
& +\varepsilon_{I}^{\Omega_{2}}\left(\boldsymbol{x}_{i}^{\in I}\right) \times\left\{\begin{array}{l}
\int_{\mathcal{H}^{\Omega_{2}+}\left(\boldsymbol{x}_{i} \in I\right)}\left(\boldsymbol{d}_{\Omega_{2}} \cdot \boldsymbol{n}_{I}^{\Omega_{2}+}\left(\boldsymbol{x}_{i}^{\in I}\right)\right) I\left(\boldsymbol{x}_{i}^{\in I}, \boldsymbol{d}_{\Omega_{2}}\right) \mathrm{d} \boldsymbol{d}_{\Omega_{2}} \\
-\sigma \times\left(4 T_{\text {ref }}^{3} T\left(\boldsymbol{x}_{i}^{\in I}\right)-3 T_{\text {ref }}^{4}\right)
\end{array}\right. \\
& +\dot{q}_{s r c}^{2 D}\left(\boldsymbol{x}_{i}^{\in I}\right)
\end{aligned}
$$

Before transforming this model into a probabilistic one and in order to get a smaller equation, we introduce $\partial\left(\boldsymbol{x}_{i}^{\in I}\right)$, which can be called a thermal dissipation coefficient, defined in Eq. (11).

$$
\partial\left(\boldsymbol{x}_{i}^{\in I}\right)=\left\{\begin{array}{l}
\frac{\lambda_{\Omega_{1}}}{\left\|\boldsymbol{x}_{i}^{\in I}-\boldsymbol{y}_{\text {cond }, \Omega_{1}}\right\| \partial \Omega_{1}}+\frac{\lambda_{\Omega_{2}}}{\left\|\boldsymbol{x}_{i}^{\in I}-\boldsymbol{y}_{\text {cond, }}^{\in \partial \Omega_{2}}\right\|} \\
+h_{I}^{\Omega_{1}}\left(\boldsymbol{x}_{i}^{\in I}\right)+h_{I}^{\Omega_{2}}\left(\boldsymbol{x}_{i}^{\in I}\right) \\
+4 \sigma T_{r e f}^{3}\left(\varepsilon_{I}^{\Omega_{1}}\left(\boldsymbol{x}_{i}^{\in I}\right)+\varepsilon_{I}^{\Omega_{2}}\left(\boldsymbol{x}_{i}^{\in I}\right)\right)
\end{array}\right.
$$

Probabilistic model. Following the same procedure as previously, we get the following probabilistic model (Eq. (12)). The probabilities associated with locally irrelevant contributions become null by taking zero as default values for parameters and physical properties. Thus, this convention allows to automatically adapt this general model to all the encountered possibilities.

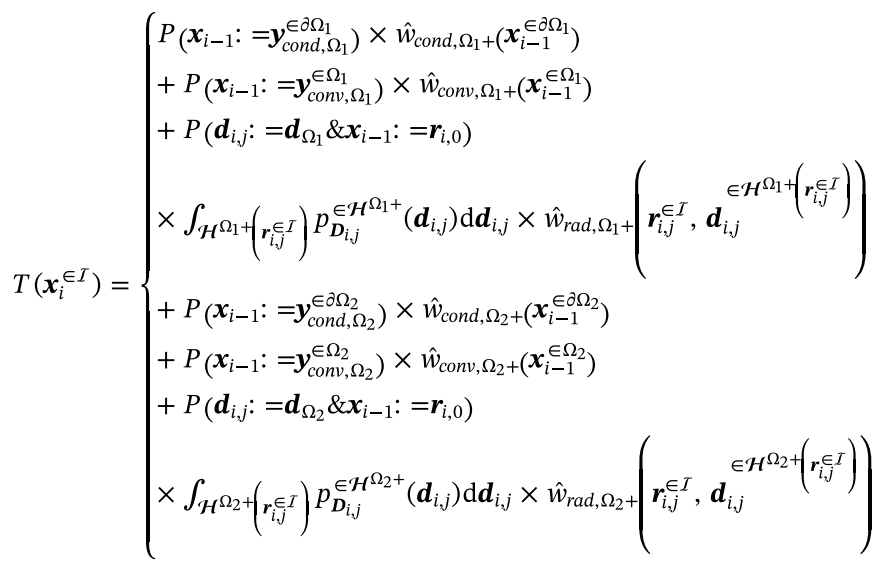

Where

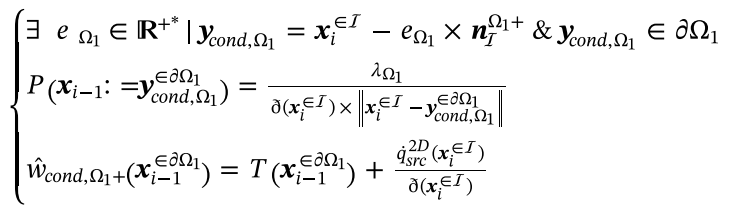

$$
\left\{\begin{array}{l}
\exists \boldsymbol{y}_{c o n v, \Omega_{1}} \in \Omega_{1} \\
P\left(\boldsymbol{x}_{i-1}:=\boldsymbol{y}_{c o n v, \Omega_{1}}^{\in \Omega_{1}}\right)=\frac{h_{I}^{\Omega_{1}}\left(\boldsymbol{x}_{i}^{\in I}\right)}{\partial\left(\boldsymbol{x}_{i}^{\in I}\right)} \\
\hat{w}_{c o n v, \Omega_{1}+}\left(\boldsymbol{x}_{i-1}^{\in \Omega_{1}}\right)=T\left(\boldsymbol{x}_{i-1}^{\in \Omega_{1}}\right)+\frac{\dot{q}_{s r c}^{2 D}\left(\boldsymbol{x}_{i} \in I\right.}{\partial\left(\boldsymbol{x}_{i}^{\in I}\right)}
\end{array}\right.
$$




$$
\begin{aligned}
& \boldsymbol{r}_{i, j}:=\boldsymbol{x}_{i}^{\in I} \\
& \exists \boldsymbol{d}_{\Omega_{1}} \in \mathcal{H}^{\Omega_{1}+}\left(\boldsymbol{r}_{i, j}^{\in I}\right) \\
& P\left(\boldsymbol{d}_{i, j}:=\boldsymbol{d}_{\Omega_{1}} \& \boldsymbol{x}_{i-1}:=\boldsymbol{r}_{i, 0}\right)=\frac{4 \varepsilon_{I}^{\Omega_{1}}\left(\boldsymbol{r}_{i, j} \in I\right) \sigma T_{r e f}^{3}}{\partial\left(\boldsymbol{r}_{i, j} \in I^{\prime}\right)}
\end{aligned}
$$

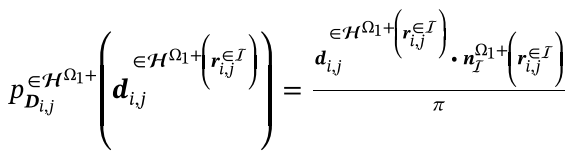

$$
\begin{aligned}
& \hat{w}_{r a d, \Omega_{1}+}\left(\boldsymbol{r}_{i, j}^{\in I}, \boldsymbol{d}_{i, j}^{\in \mathcal{H}^{\Omega_{1}+}\left(\boldsymbol{r}_{i, j}^{\in I}\right)}\right)=\frac{\left.\pi I\left(\boldsymbol{r}_{i, j}^{\in I}, \boldsymbol{d}_{i, j}^{\in \mathcal{H}^{\Omega_{1}+}+\boldsymbol{r}_{i, j} \in I}\right)\right)+3 \sigma T_{\text {ref }}^{4}}{4 \sigma T_{\text {ref }}^{3}}+\frac{\dot{q}_{s r c}^{2 D}\left(\boldsymbol{r}_{i, j}^{\in I}\right)}{\partial\left(\boldsymbol{r}_{i, j} \in I\right)}
\end{aligned}
$$

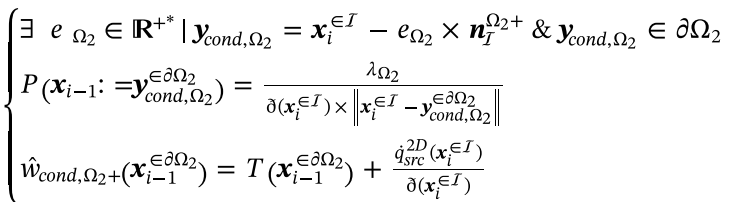

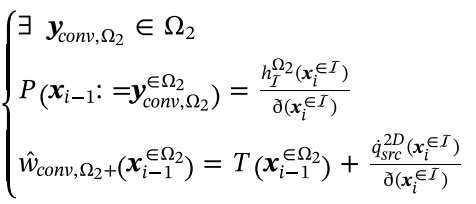

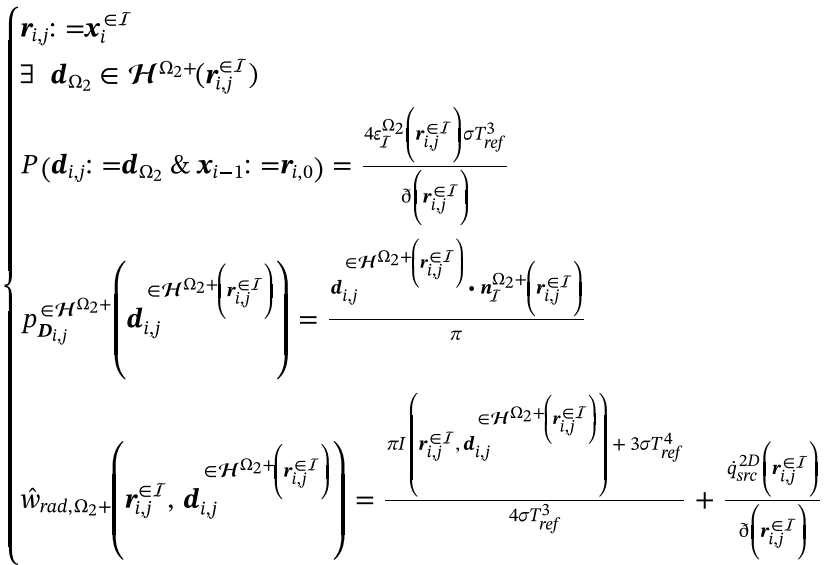

In the following section, two different models for the total directional intensity (in incident or leaving directions) are presented. These models are showing that the incident intensity is function of a leaving intensity at another position (Eq. (13)), which can be itself linked either to the temperature field (by the radiation emission phenomenon) or to the incident intensity field (by reflection for example) (Eq. (14)). Thus, the evaluation of the incident intensity model requires to construct an other exploration path (where intensity models are recursively evaluated) to find the emission location which is the next point in the temperature exploration path. In order to distinguish them, the $\boldsymbol{r}_{i, j}$ positions are used for the intensity path starting at the $\boldsymbol{x}_{i}$ point. This complementary path can be seen as an intricate sampling function of the next temperature path position $\left(\boldsymbol{x}_{i-1}:=\boldsymbol{r}_{i, 0}\right)$. Thus, with our systemic point of view, the radiative transfer has exactly the same impact on the temperature exploration path as the other heat transfer phenomena.

\subsection{Field of total directional intensity incident at an interface}

The total directional intensity is defined as incident from the $\Omega$ domain at the point $\boldsymbol{r}_{i, j}$ on the domain boundary when its direction is in the hemisphere $\mathcal{H}^{\Omega+}\left(\boldsymbol{r}_{i, j}^{\in \partial \Omega}\right)$ (Fig. 7). Domains can either be opaque or totally transparent. In the former case, no light is spreading through the domain and the field of total directional intensity incident is null. In the latter case, the light is transmitted without any interaction with the domain. Hence, the total directional intensity is constant along its direction into the domain and only the interfaces are exchanging by radiative transfer. Consequently, with $\boldsymbol{r}_{i, j-1}^{\in \partial \Omega}$ defined as the intersection point of a ray leaving the point $\boldsymbol{r}_{i, j}^{\in \partial \Omega}$ in the direction $-\boldsymbol{d}_{i, j}^{\in \mathcal{H}^{\Omega+}}\left(\boldsymbol{r}_{i, j}^{\in \partial \Omega}\right)$ with the domain boundary $\partial \Omega$, we have the following model (Eq. (13)):

$I\left(\boldsymbol{r}_{i, j}^{\in \partial \Omega}, \boldsymbol{d}_{i, j}^{\in \mathcal{H}^{\Omega+}\left(r_{i, j}^{\in \partial \Omega}\right)}\right)=I\left(\boldsymbol{r}_{i, j-1}^{\in \partial \Omega}, \boldsymbol{d}_{i, j}^{\in \mathcal{H}^{\Omega-}\left(r_{i, j-1}^{\in \partial \Omega}\right)}\right)$

With

$\exists e_{j} \in \mathbb{R}^{+*} \mid \boldsymbol{r}_{i, j-1}=\boldsymbol{r}_{i, j}^{\in \partial \Omega}-e_{j} \times \boldsymbol{d}_{i, j}^{\in \mathcal{H}^{\Omega+}\left(\boldsymbol{r}_{i, j}^{\in \partial \Omega}\right)} \& \boldsymbol{r}_{i, j-1} \in \partial \Omega$

This model has a single contribution. Thus its deterministic form is fully equivalent to a probabilistic form with a probability of 1 of continuing the path with the single contribution.

\subsection{Field of total directional intensity leaving an interface}

Deterministic model. The total directional intensity leaving the point $\boldsymbol{r}_{i, j-1}^{\in \partial \Omega}$ in the direction $\boldsymbol{d}_{i, j}^{\in \mathcal{H}^{\Omega-}\left(\boldsymbol{r}_{i, j-1}^{\in \neq \Omega}\right)}$ is the sum of potentially three phenomena (Eq. (14) and Figs. 8-11): emission, reflection (with a specular part defined by $s_{\partial \Omega}^{\Omega}$ ) or transmission by the interface.

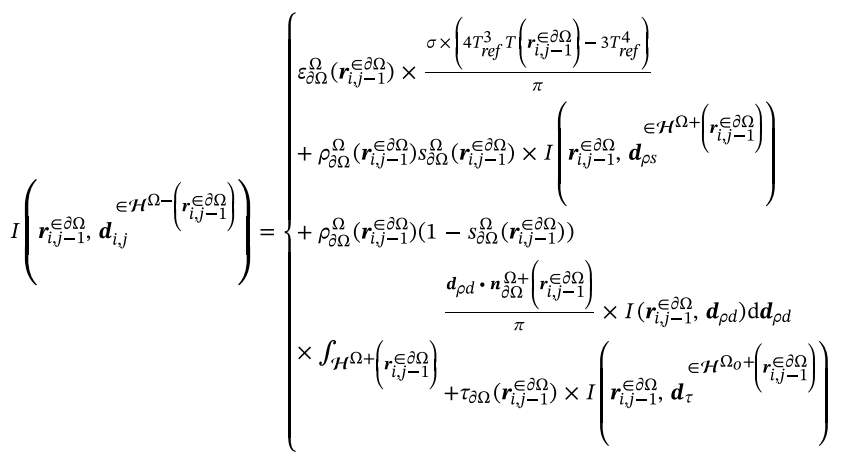

In Eq. (14), we can see two directions preceding the reflection phenomena. The first one is associated to the diffuse reflection:

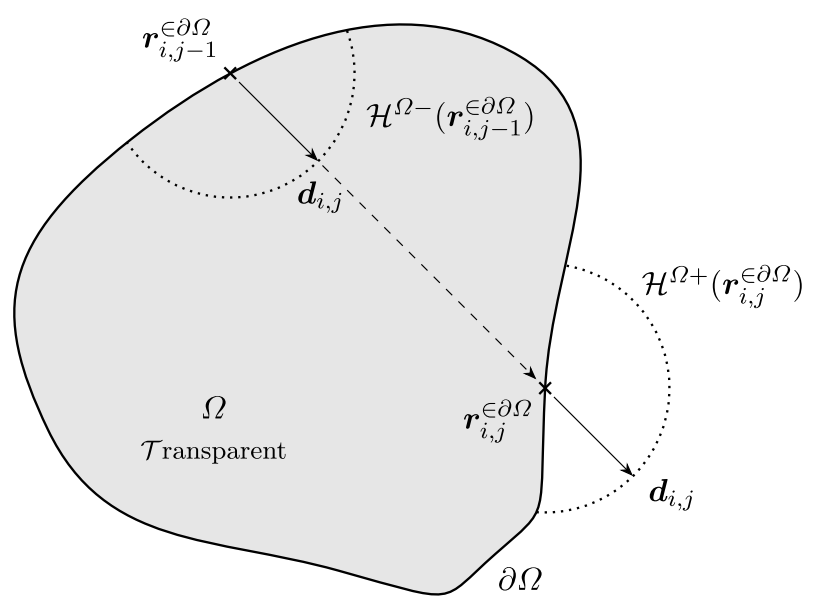

Fig. 7. Diagram representing the notations used in the total directional intensity model for directions towards an interface. 


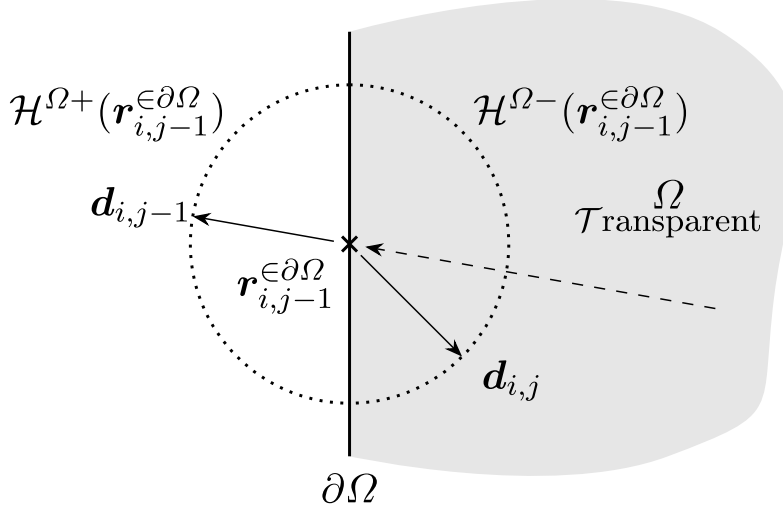

Fig. 8. Diagram representing the notations used in the total directional intensity model for directions leaving an interface by a diffuse reflection.

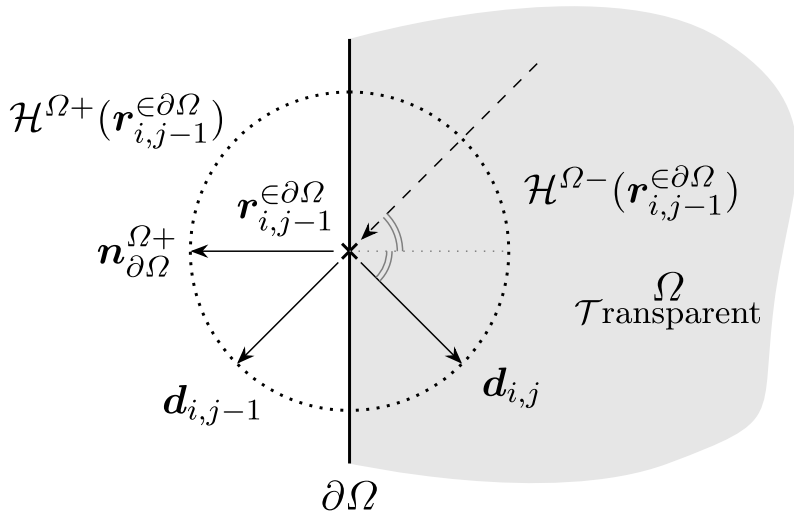

Fig. 9. Diagram representing the notations used in the total directional intensity model for directions leaving an interface by a specular reflection.

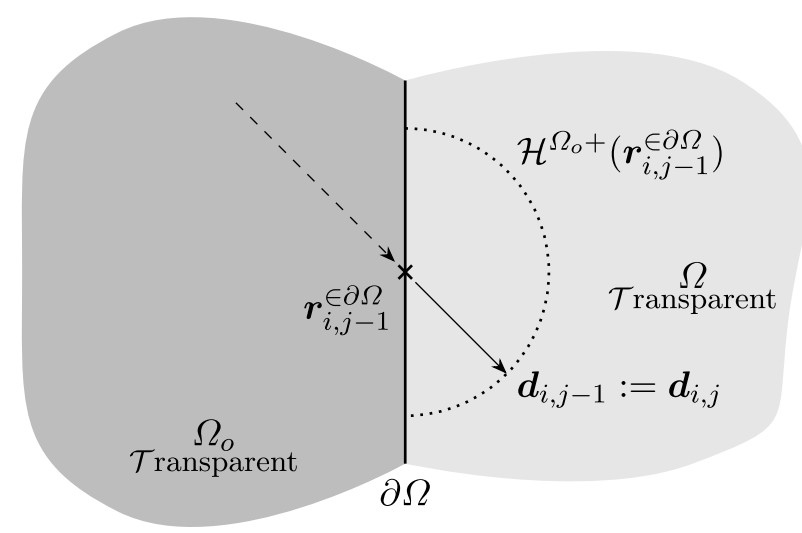

Fig. 10. Diagram representing the notations used in the total directional intensity model for directions leaving an interface by transmission.

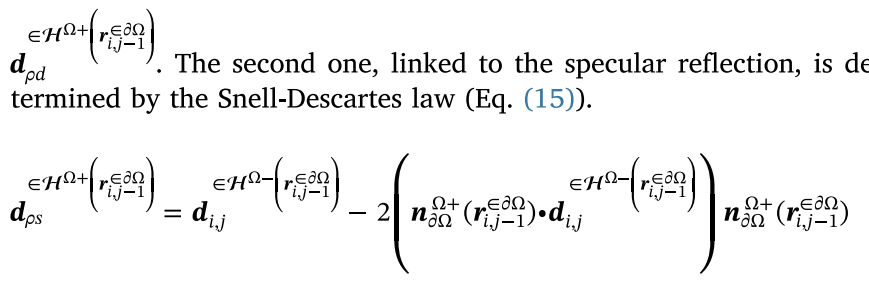

Probabilistic model. Thanks to the Kirchhoff law of thermal radiation, we can rearrange it to get the equivalent probabilistic model (Eq. (16)).

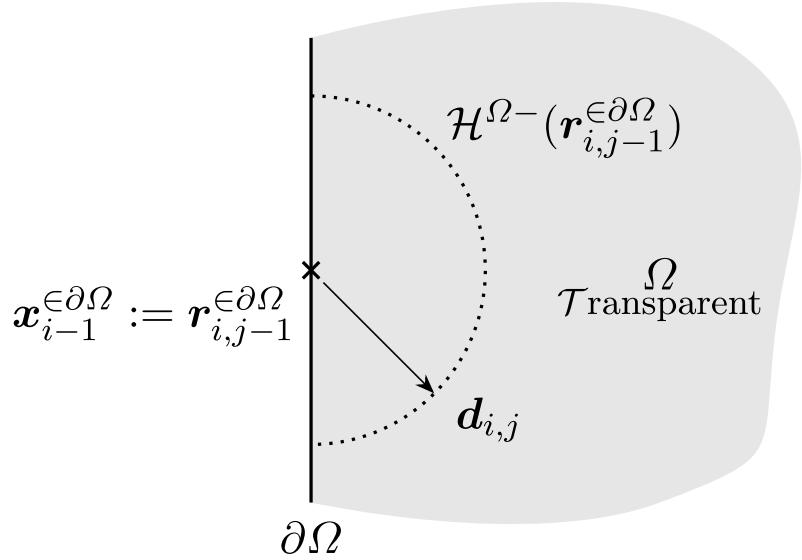

Fig. 11. Diagram representing the notations used in the total directional intensity model for directions leaving an interface by emission.

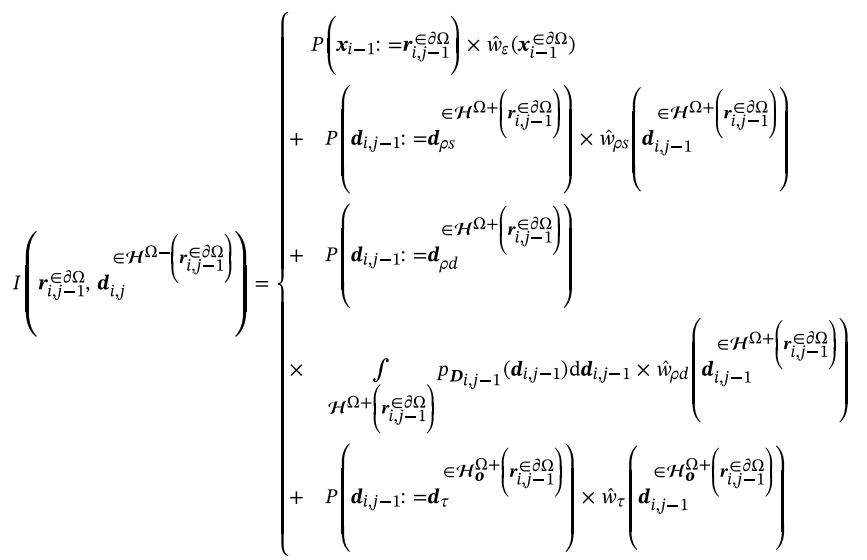

Where

$\left\{\begin{array}{l}P\left(\boldsymbol{x}_{i-1}:=\boldsymbol{r}_{i, j-1}^{\in \partial \Omega}\right)=\varepsilon_{\partial \Omega}^{\Omega}\left(\boldsymbol{r}_{i, j-1}^{\in \partial \Omega}\right) \\ \hat{w}_{\varepsilon}\left(\boldsymbol{x}_{i-1}^{\in \partial \Omega}\right)=\frac{\sigma \times\left(4 T_{r e f}^{3} T\left(\boldsymbol{x}_{i-1}^{\in \partial \Omega}\right)-3 T_{r e f}^{4}\right)}{\pi}\end{array}\right.$

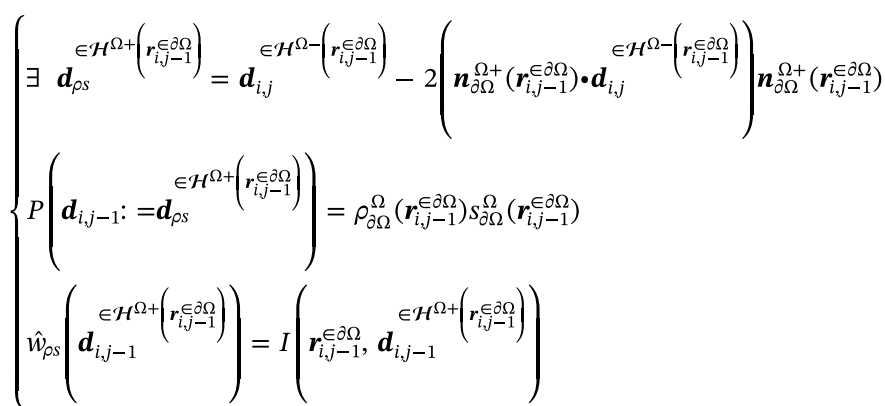

$$
\begin{aligned}
& \left(\begin{array}{l}
\exists \boldsymbol{d}_{\rho d} \in \mathcal{H}^{\Omega+}\left(\boldsymbol{r}_{i, j-1}^{\in \partial \Omega}\right) \\
P\left(\begin{array}{c}
\left.\boldsymbol{d}_{i, j-1}:=\boldsymbol{d}_{\rho d}^{\in \mathcal{H}^{\Omega+}\left(\boldsymbol{r}_{i, j-1}^{\in \partial \Omega}\right)}\right) \\
\end{array}\right)=\rho_{\partial \Omega}^{\Omega}\left(\boldsymbol{r}_{i, j-1}^{\in \partial \Omega}\right)\left(1-s_{\partial \Omega}^{\Omega}\left(\boldsymbol{r}_{i, j-1}^{\in \partial \Omega}\right)\right)
\end{array}\right.
\end{aligned}
$$

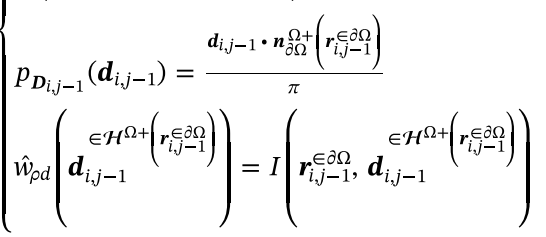




$$
\begin{aligned}
& \int \boldsymbol{d}_{\tau}^{\in \mathcal{H}^{\Omega_{0}+}\left(\boldsymbol{r}_{i, j-1} \in \Omega_{1}\right)}=\boldsymbol{d}_{i, j}^{\in \mathcal{H}^{\Omega_{0}+}\left(\boldsymbol{r}_{i, j-1} \in \Omega_{1}\right)} \\
& \left\{P\left(\boldsymbol{d}_{i, j-1}:=\boldsymbol{d}_{\tau}^{\in \mathcal{H}^{\Omega_{o}+}\left(\boldsymbol{r}_{i, j-1}^{\in \partial \Omega}\right)}\right)=\tau_{\partial \Omega}\left(\boldsymbol{r}_{i, j-1}^{\in \partial \Omega}\right)\right. \\
& \hat{w_{\tau}}\left(\begin{array}{c}
\in \boldsymbol{d}_{i, j-1}^{\Omega_{o}+}\left(\boldsymbol{r}_{i, j-1}^{\in \partial \Omega}\right) \\
\end{array}\right)=I\left(\boldsymbol{r}_{i, j-1}^{\in \partial \Omega}, \boldsymbol{d}_{i, j-1}^{\in \mathcal{H}^{\Omega_{O}+}\left(\boldsymbol{r}_{i, j-1}^{\in \partial \Omega}\right)}\right)
\end{aligned}
$$

\subsection{Validation}

This probabilistic modeling framework has then been validated against analytical results of 13 academic test cases. Firstly, 9 cases illustrating a single phenomenon of thermal heat transfer (conduction, convection or radiation) have been tested. In these cases the temperature field at several points has been estimated and showed a perfect agreement with the analytical expectations. Secondly, 4 additional cases, where at least two transfer modes are coupled together, have been undertaken. These tests have also been successful. All of this allows us to be very confident in the framework results and to consider them as reference results in validity domain of the aforementioned taken assumptions. Some of these validation cases are provided as supplementary materials.

\section{Application and discussion}

\subsection{Application case: the Building Module}

Some applications cases in the thermal simulation of buildings have been chosen to illustrate the framework capabilities. Problems of increasing geometrical complexity have been built using a Building Module as the constituent sub-system (Fig. 12). They are spread from a single Building Module up to a compound of 50 of them. Each Building Module consists of 7 domains separated by interfaces ( 5 walls, a glazed window and the inner air). In addition, the external environment is taken into account.

Walls. The five walls are $0.2 \mathrm{~m}$ thick with a thermal conductivity of $0.12 \mathrm{~W} . \mathrm{m}^{-1} . \mathrm{K}^{-1}$ in order to model autoclaved aerated concrete [39]. The ground floor is considered adiabatic. The inner and outer walls have an 0.5 emissivity completed with a totally diffuse reflection. Below the window, an area of $2 \times 1 \mathrm{~m}$ experiences a heat source of $700 \mathrm{~W} \cdot \mathrm{m}^{-2}$ to represent a heater.

Glazed window. The glazed window is modeled by a $5 \mathrm{~mm}$ thick piece of glass of $1.2 \mathrm{~W} \cdot \mathrm{m}^{-1}$. $\mathrm{K}^{-1}$ of thermal conductivity. Concerning its radiative properties, it is seen as perfectly opaque to infrared radiation with an emissivity of 1 .

Inner air. The inner air volume has a specific heat capacity of $1006 \mathrm{~J} . \mathrm{kg}^{-1} \cdot \mathrm{K}^{-1}$ (300 $\mathrm{K}$ and atmospheric pressure). On one hand, this volume exchanges heat with the inner side of the walls and the glazed window thanks to a convection coefficient of $5.0 \mathrm{~W} . \mathrm{m}^{-2} \cdot \mathrm{K}^{-1}$. On the other hand, the regulation air renewal is modeled by a mass flow of $8.0 \times 10^{-3} \mathrm{~kg} . \mathrm{s}^{-1}(0.5$ of the total air mass per hour) from and towards the environment.

Environment. Finally the environment has a constant temperature of $273 \mathrm{~K}$. The outer side of the walls and the window is experiencing a convection coefficient of $40.0 \mathrm{~W} \cdot \mathrm{m}^{-2} \cdot \mathrm{K}^{-1}$ with the air environment to account for windy conditions. Regarding the radiative transfer, the ground and the horizon are represented as black bodies.

In all these cases, the Monte Carlo simulations aim at estimating the inner air temperature of a Building Module located around the scene center.

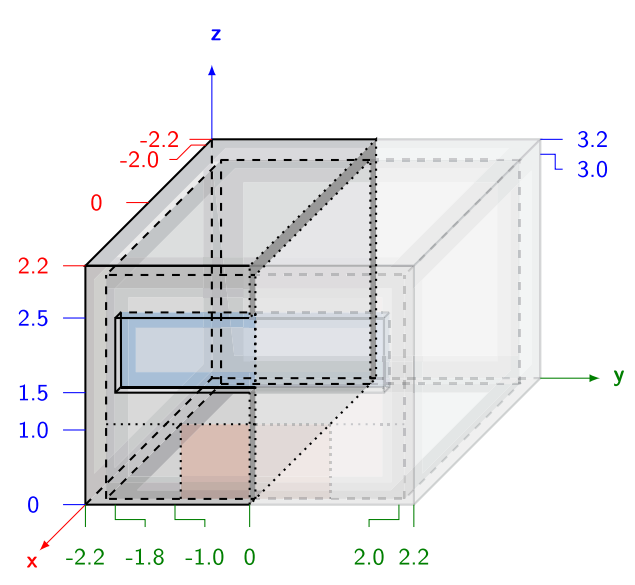

Fig. 12. Cross-sectional representation of a Building Module.

\subsection{Results and discussion}

In order to assess these models, we have used the open source EDStaR framework, which is a dedicated tool to build and run Monte Carlo algorithms in complex systems [40,41].

Thermal conductivity influence. The thermal conductivity influence on the inner air temperature of a single Building Module has been investigated (Table 1 and Fig. 13). Inner temperature is firstly falling from $302.21 \mathrm{~K}$ (for a conductivity of $0.04 \mathrm{~W} \cdot \mathrm{m}^{-1} \cdot \mathrm{K}^{-1}$ ) to $276.75 \mathrm{~K}$ (at $1.20 \mathrm{~W} . \mathrm{m}^{-1} \cdot \mathrm{K}^{-1}$ ) and then decreases slowly until to converge at a $273 \mathrm{~K}$ asymptote. When the walls have a low thermal conductivity, the heat conduction through them is the limiting phenomenon in terms of heat transfer. This explains why increasing the wall capability to transfer heat has an significant impact on the building heat losses. From a thermal conductivity of $1.0 \mathrm{~W} . \mathrm{m}^{-1} . \mathrm{K}^{-1}$ and higher, the inner convection becomes the limiting transfer phenomenon, which explains why increasing thermal conductivity further has almost no influence. The previously described behavior could be expected from common heat engineering knowledge. Our framework was able to capture it without any human intervention, which is a token of its quality.

Computational time assessment. The Monte Carlo method enables an easy parallelization of the computation by allowing each process to independently explore the scene. Nevertheless, the computational time have been assessed without using it, on a mainstream computer (CPU: Intel Core i5-2435M (Sandy Bridge) $2.4 \mathrm{GHz} \mid$ RAM: 4 Go DDR3 1333 Mhz). For Monte Carlo simulations, the number of histories, and hence the run time, is linked with the standard error of the obtained result. That is why, as an indicator, we chose to estimate the computational time needed to reach a standard error of $0.1 \%$ on the temperature estimation in this work. In order to present more reliable computational time data, a batch of 12 runs for each case have been done and the two extrema have been removed before the estimation of mean value and standard error.

Dependence on the exploration path length. An estimation by the Monte Carlo algorithm consists in creating a great number of exploration paths. Hence, the lengths of these paths play a part in its execution time. They have been defined as the number of evaluation points between the probe and the final explicit data location. To assess the impact of this variable, the mean lengths $(\langle i+j\rangle)$ of paths originating from each probe have been estimated. The plot of the computational time evolution against this variable (Fig. 14) shows that the former is clearly correlated to the mean exploration path length $\left(R^{2}=0.985\right)$.

Asymptotic exploration. On one hand, the mean exploration path length starts from 22 evaluation points for a single module and is converging to 31 evaluation points for scenes of ten modules and more (Fig. 15 and Table 2). According to the aforementioned correlation, the 
execution time shows the same asymptotic trend with $1.0 \mathrm{~s}$ to compute the first scene and an asymptote value around $2.1 \mathrm{~s}$. In other words, the addition of Building Modules in the scene does not significantly increase the computational time although the problem becomes much more complex and extensive.

Problem influence area. On the other hand, the air temperature value estimated inside the central Building Module presents also an asymptotic trend. Actually, when the Building Module is alone, its inner air has a temperature of $292.16 \mathrm{~K}$ (Table 2). By adding three other modules, one above and the two others on sides, this temperature increases to $296.92 \mathrm{~K}$. Finally, the temperature is converging towards approximately $300 \mathrm{~K}$ when the number of Building Modules involved in the scene increases. The magnitude of the first increase can be explained by the fact that adding modules improves the insulation of the first one. However, as the additional modules are more and more distant from the probe location, they have less and less influence on the inner air temperature of the first module, which explains the noticed asymptotic trend.

Probability value effect. These two facts are related by the probability values used in the models. Indeed, by choosing them to be the closest as possible to the physical phenomena described, the exploration paths are directed towards the system parts influencing the probe. Applied to the heat transfer physics, this means choosing probability value of each exchange contribution proportionally to its heat transfer capacity. This approach explains also why the Monte Carlo weights associated with each contribution in the models are generally a simple expression of the physical field at an other location. Actually, when linear models are used, the heat transfer intensity results from a difference in the thermal potential field (temperature or radiative intensity) flowing trough a heat transfer capacity (by multiplication).

Self-adaptive computation. All of this means that the computational time of this approach is depending almost exclusively on the influential parts of the problem, which are automatically identified in a selfadaptive way. Hence, the computational time does not depend on the whole scene complexity. This is a major beneficial behavior for the complex system simulation and their optimization. Indeed, by definition of these systems, it is very difficult for the user to determine $a$ priori which part of the system has an important influence on the probe, or on the objective function estimation, and which has not. In addition, during an optimization process, identifying the influential parts of the problem to be modeled, for each assessed proposal, is commonly a timeconsuming step even for rather simple systems. This self-adaptive estimation algorithm allows now the user to save this pre-processing time.

Comparison with grid-based deterministic approach. Logically, this result on computational time have to be compared with those obtainable by a grid-based deterministic approach. However, a such unbiased comparison is not straightforward due to the great heterogeneity existing among these two kinds of methods (assumptions, parameters and result structures). On one side, the grid-based approach computes a field estimation, at each grid element, resulting in a runtime complexity almost proportional to the discretizing elements number for the best algorithms. On the other side, the Monte Carlo algorithm estimates the field at each positioned probe in the system by the number of stochastic histories enabling to end up with the aimed statistical precision; each history consisting in recursively building exploration paths in the present proposal. Thereby, its runtime complexity is proportional to the number of probes and evaluation histories used, multiplied by the mean path length, which essentially depends on the probability value choices and on the probe locations. Consequently, direct comparison is not

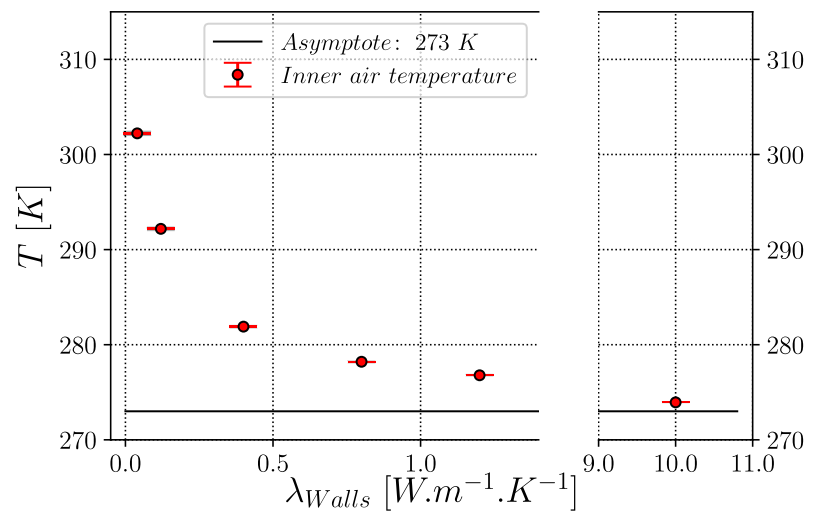

Fig. 13. Graph representing the inner air temperature evolution for a single Building Module against its wall thermal conductivity.

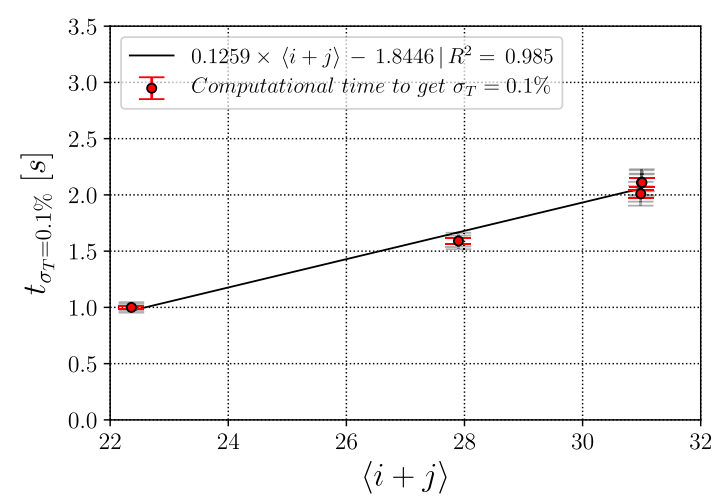

Fig. 14. Graph representing the computational time evolution, on a single processor thread, against the mean exploration path lengths.

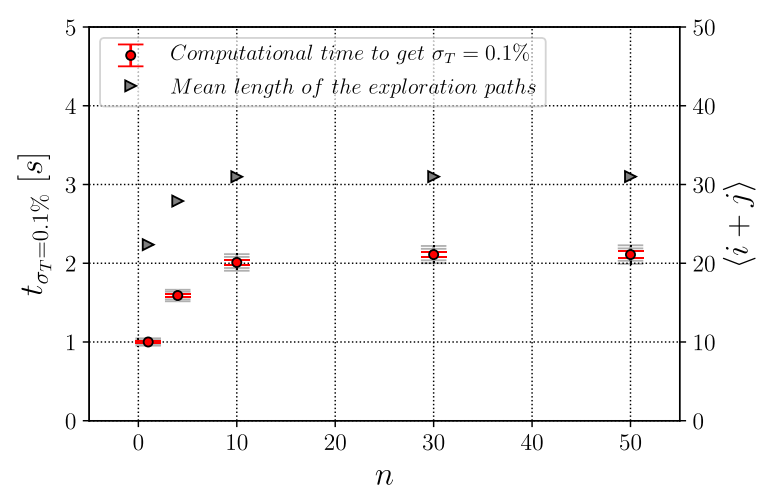

Fig. 15. Graph representing the mean exploration path length and the computational time evolutions, on a single processor thread, against the number of Building Modules in the simulated scene.

possible. Deterministic grid-based methods yet appear to be the most efficient choice in some cases: low complexity systems, problems with at most three integral dimensions or involving non-linear phenomena,

Table 1

Table of the estimated temperatures, and their standard errors, inside a single Building Module depending on the thermal conductivity of the walls.

\begin{tabular}{llllllll}
\hline$\lambda_{\text {Walls }}\left[W . m^{-1} \cdot K^{-1}\right]$ & 0.040 & 0.12 & 0.40 & 0.80 & 1.2 & 3.0 & 5.0 \\
\hline$T[K]$ & 302.21 & 292.16 & 281.86 & 278.18 & 276.75 & 274.86 & 274.33 \\
$\sigma_{T}\left[10^{-3} K\right]$ & 59.7 & 45.1 & 27.1 & 18.8 & 15.0 & 9.17 & 7.27 \\
\hline
\end{tabular}


Table 2

Table of the estimated temperatures, the needed computational times (and their standard errors) and the mean lengths of exploration paths depending on the number of Building Modules in the simulated scene.

\begin{tabular}{llllll}
\hline$n$ & 1 & 4 & 10 & 30 & 50 \\
\hline$T[K]$ & 292.16 & 296.92 & 299.43 & 299.45 & 299.46 \\
$\left.\left\langle t_{\sigma_{T}=0.1 \%}\right\rangle s\right]$ & 1.00 & 1.59 & 2.01 & 2.11 & 2.11 \\
$\sigma_{t}\left[10^{-2} s\right]$ & 1.55 & 2.44 & 3.52 & 3.65 & 3.93 \\
$\langle i+j\rangle$ & 22.36 & 27.90 & 30.99 & 31.00 & 31.01 \\
\hline
\end{tabular}

or even when the whole physical field estimation is needed. To tackle problems with opposite characteristics, the Monte Carlo method appears more relevant. In the end, there are different tools fitted for different problems.

\section{Conclusion}

In this work, we have presented a modular framework which allows to construct probabilistic models of coupled heat transfer cases. A systemic approach has been implemented in order to substructure problems in assembled physical field submodels.

First, deterministic submodels of the physical fields in different component classes have been established. Each model dealing with one phenomenon of heat transfer, or more, i.e. conduction, convection and radiation. Then, these models have been rewritten from the deterministic point of view to a probabilistic one. This enabled us to solve the model combination using a unique recursive Monte Carlo algorithm. Next, the framework results were successfully validated against analytical results. Finally, it was applied to tackle an elaborated toy problem emulating heat transfer in a building.

Over the course of this paper, the framework has demonstrated its capability to find classic behaviors such as identifying the limiting heat transfer phenomenon in a problem. In addition to that, taking full advantage of a Monte Carlo method faithful to physical phenomena, this approach has shown to be able to select the most influential parts of the problem on its own. Hence, its need for computational power is reduced to what is required to only explore the contributing parts of the domain. This enables the algorithm to tackle multiscale problems, with complicated and/or extensive geometry.

To put it in a nutshell, the framework computational speed is not hindered by the complexity and/or extensiveness of the problem as it selects all by itself the relevant fractions of the domain making it a selfadaptive simulation method. Furthermore, a recent work [42] established that this modeling approach enables to end up with estimations not only as values but also as functions of the boundary conditions, by simplifying the Symbolic Monte Carlo algorithm application inside complex systems. These behaviors appear as major benefits to enable engineers and designers to simulate and optimize increasingly complex systems.

\section{Appendix A. Supplementary data}

Supplementary data related to this article can be found at http://dx. doi.org/10.1016/j.ijthermalsci.2018.04.004.

\section{References}

[1] G.G. Wang, S. Shan, Review of metamodeling techniques in support of engineering design optimization, J Mech Des 129 (4) (2007) 370, http://dx.doi.org/10.1115/1. 2429697 ISSN 10500472, doi:\bibinfo.

[2] A.-T. Nguyen, S. Reiter, P. Rigo, A review on simulation-based optimization methods applied to building performance analysis, Appl Energy 113 (2014) 1043-1058, http://dx.doi.org/10.1016/j.apenergy.2013.08.061 ISSN 03062619, doi: \bibinfo http://www.sciencedirect.com/science/article/pii/ S0306261913007058.

[3] R.T. Marler, J.S. Arora, Survey of multi-objective optimization methods for engineering, Struct Multidiscip Optim 26 (6) (2004) 369-395, http://dx.doi.org/ 10.1007/s00158-003-0368-6 ISSN 1615147X, doi:\bibinfo.

[4] N. Metropolis, S. Ulam, The Monte Carlo method, (1949), http://dx.doi.org/10. 1080/01621459.1949.10483310 doi: \bibinfo http://www.tandfonline.com/doi/ abs/10.1080/01621459.1949.10483310.

[5] J. Hammersley, D. Handscomb, Monte Carlo methods, Wiley, 1964.

[6] R.E. Caflisch, Monte Carlo and quasi-Monte Carlo methods, (1998), http://dx.doi. org/10.1017/S0962492900002804 doi:\bibinfo.

[7] K. Mosegaard, M. Sambridge, Monte Carlo analysis of inverse problems, Inverse Probl 18 (3) (2002) R29-R54, http://dx.doi.org/10.1088/0266-5611/18/3/201 ISSN 0266-5611, doi: \bibinfo.

[8] W.L. Dunn, J.K. Shultis, Exploring Monte Carlo methods, Academic Press, 2012 ISBN 9780444515759.

[9] F.J. Hickernell, H.S. Hong, P. L'Écuyer, C. Lemieux, Extensible lattice sequences for Quasi-Monte Carlo quadrature, SIAM J Sci Comput 22 (3) (2000) 1117-1138, http://dx.doi.org/10.1137/S1064827599356638 ISSN 1064-8275, doi:`bibinfo http://epubs.siam.org/doi/abs/10.1137/S1064827599356638.

[10] F.B. Brown, W.R. Martin, R.D. Mosteller, Monte Carlo - advances and challenges, Tech. Rep. Los Alamos National Laboratory, 2008, http://dx.doi.org/10.1039/ c4ta02097a doi: \bibinfo.

[11] J. Dauchet, Analyse radiative des photobioréacteurs, Ph.d. thesis Université Blaise Pascal - Clermont II, 2012.

[12] O. Farges, Conception optimale de centrales solaires a concentration : application aux centrales a tour et aux installations "beam down", Ph.d. thesis Mines Albi, 2015.

[13] G. Terrée, Méthode de Monte-Carlo et non-linéarités: de la physique du transfert radiatif à la cinétique des gaz, Ph.d. thesis Mines Albi, 2015.

[14] J.G. Michopoulos, C. Farhat, J. Fish, Modeling and simulation of multiphysics systems, Comput Inf Sci Eng 5 (2005) 198-213, http://dx.doi.org/10.1115/1. 2031269 ISSN 00092479, doi: $\backslash$ bibinfo.

[15] S. Portegies Zwart, S. McMillan, S. Harfst, D. Groen, M. Fujii, B.O. Nuallain, et al., A multiphysics and multiscale software environment for modeling astrophysical systems, New Astron 14 (4) (2009) 369-378, http://dx.doi.org/10.1016/j.newast. 2008.10.006 ISSN 13841076, doi: \bibinfo.

[16] G.-W. Wei, Multiscale, Multiphysics and Multidomain Models I: Basic Theory, J Theor Comput Chem 12(08), ISSN 0219-6336, doi: \bibinfo \{doi\} \{https://10.1142/ S021963361341006X\}.

[17] D. a. Kessler, E.S. Oran, C.R. Kaplan, Towards the development of a multiscale, multiphysics method for the simulation of rarefied gas flows, J Fluid Mech 661 (2010) 262-293, http://dx.doi.org/10.1017/S0022112010002934 ISSN 00221120, doi: \bibinfo http://www.journals.cambridge.org/abstract $\% 7 b \% 5 c$ $\% 7$ dS0022112010002934.

[18] T. Tautges, H. Kim, A. Caceres, R. Jain, Coupled Multi-Physics simulation frameworks for reactor simulation: a Bottom-Up approach, Int. Conf. Math. Comput. Methods appl. To nucl. Sci. Eng, 2011 ISBN 9788563688002 http:// mathematicsandcomputation.cowhosting.net/MC11/\%7b $\% 5 c_{-} \% 7 \mathrm{dpdf} / 0 \% 7 \mathrm{~b} \% 5 \mathrm{c}_{-}$ $\% 7 d 20101231122229 \% 7 b \% 5 c \_\% 7 d p a p e r . p d f$.

[19] Taik Young Kim, Seung Wook Baek, Analysis of combined conductive and radiative heat transfer in a two-dimensional rectangular enclosure using the discrete ordinates method, Int J Heat Mass Transf 34 (9) (1991) 2265-2273, http://dx.doi.org/ 10.1016/0017-9310(91)90052-G ISSN 00179310, doi:\bibinfo.

[20] M.Y. Kim, Seung Wook Baek, Numerical analysis of conduction, convection, and radiation in a gradually expanding channel, Numer Heat Transf Part A Appl An Int J Comput Methodol 29 (7) (1996) 725-740, http://dx.doi.org/10.1080/10407782. 2011.552363 doi: $\backslash$ bibinfo.

[21] D. Lacroix, G. Parent, F. Asllanaj, G. Jeandel, Coupled radiative and conductive heat transfer in a non-grey absorbing and emitting semitransparent media under collimated radiation, J Quant Spectrosc Radiat Transf 75 (5) (2002) 589-609, http://dx doi.org/10.1016/S0022-4073(02)00031-6 ISSN 00224073, doi: \bibinfo.

[22] F. Asllanaj, G. Jeandel, J.R. Roche, D. Lacroix, Transient combined radiation and conduction heat transfer in fibrous media with temperature and flux boundary conditions, Int J Therm Sci 43 (10) (2004) 939-950 ISSN 12900729.

[23] S. Mahapatra, P. Nanda, A. Sarkar, Analysis of coupled conduction and radiation heat transfer in presence of participating medium- using a hybrid method, Heat Mass Transf 41 (10) (2005) 890-898, http://dx.doi.org/10.1007/s00231-0040587-4 ISSN 0947-7411, doi:\bibinfo http://link.springer.com/10.1007/s00231004-0587-4.

[24] L.M. Ruan, M. Xie, H. Qi, W. An, H.P. Tan, Development of a finite element model for coupled radiative and conductive heat transfer in participating media, J Quant Spectrosc Radiat Transf 102 (2) (2006) 190-202, http://dx.doi.org/10.1016/j.jqsrt. 2006.02.070 ISSN 00224073, doi: \bibinfo.

[25] K.A.R. Ismail, C.T.S. Salinas, Gray radiative conductive 2D modeling using discrete ordinates method with multidimensional spatial scheme and non-uniform grid, Int $\mathrm{J}$ Therm Sci 45 (7) (2006) 706-715, http://dx.doi.org/10.1016/j.ijthermalsci.2005. 10.004 ISSN 12900729 , doi: \bibinfo.

[26] H. Amiri, S.H. Mansouri, A. Safavinejad, Combined conductive and radiative heat transfer in an anisotropic scattering participating medium with irregular geometries, Int J Therm Sci 49 (3) (2010) 492-503, http://dx.doi.org/10.1016/j. ijthermalsci.2009.10.005 ISSN 12900729, doi: \bibinfo https://doi.org/10.1016/j. ijthermalsci.2009.10.005.

[27] A. Al Abed, J.-F. Sacadura, A Monte Carlo-Finite difference method for coupled radiation-conduction heat transfer in semitransparent media, J Heat Transfer 105 (1983) 931-933.

[28] H. Schweiger, A. Oliva, M. Costa, C.D. Perez Segarra, A Monte Carlo method for the simulation of transient radiation heat transfer: application to compound honeycomb transparent insulation, Numer Heat Transf Part B Fundam 35 (1999) 
$113-136$.

[29] S.W. Baek, D.Y. Byun, S.J. Kang, The combined Monte-Carlo and finite-volume method for radiation in a two-dimensional irregular geometry, Int $\mathrm{J}$ Heat Mass Transf 43 (13) (2000) 2337-2344, http://dx.doi.org/10.1016/S0017-9310(99) 00288-4 ISSN 00179310, doi: \bibinfo.

[30] Z. Cheng, Y. He, F. Cui, Numerical investigations on coupled heat transfer and synthetical performance of a pressurized volumetric receiver with MCRT-FVM method, Appl Therm Eng 50 (1) (2013) 1044-1054, http://dx.doi.org/10.1016/j applthermaleng.2012.08.045 ISSN 13594311, doi:\bibinfo http://linkinghub. elsevier.com/retrieve/pii/S1359431112005777.

[31] A.E. Kovtanyuk, A.Y. Chebotarev, An iterative method for solving a complex hea transfer problem, Appl Math Comput 219 (17) (2013) 9356-9362, http://dx.doi. org/10.1016/j.amc.2013.03.091 ISSN 00963003, doi:\bibinfo http://linkinghub. elsevier.com/retrieve/pii/S0096300313003524.

[32] R. Fournier, S. Blanco, V. Eymet, M. El Hafi, C. Spiesser, Radiative, conductive and convective heat-transfers in a single Monte Carlo algorithm, J Phys Conf Ser 676 (2016) 1-3, http://dx.doi.org/10.1088/1742-6596/676/1/012007 ISSN 17426588, doi: \bibinfo.

[33] G.L. Vignoles, A hybrid random walk method for the simulation of coupled conduction and linearized radiation transfer at local scale in porous media with opaque solid phases, Int J Heat Mass Transf 93 (2016) 707-719, http://dx.doi.org/10. 1016/j.ijheatmasstransfer.2015.10.056 ISSN 00179310, doi:\bibinfo http:// linkinghub.elsevier.com/retrieve/pii/S0017931015010388.

[34] J.-M. Nataf, R. Ebert, On the efficiency of partitioning in object-based simulation mathematical methods, Build Simulation, vol. 93, 1993, pp. 259-265 http://www ibpsa.org/proceedings/BS1993/BS93\%7b\%5c_\%7d259\%7b\%5c_\%7d265.pdf.
[35] R. Ebert, Developpement d'un environnement de simulation de systèmes complexes. Application aux bâtiments, Ph.d. thesis Ecole Nationale des Ponts et Chaussées, 1993, https://tel.archives-ouvertes.fr/tel-00523619.

[36] G. Lefebvre, E. Palomo, M.M. Izquierdo, Reproducing thermal coupling between components in a generic environment like matlab, Proceedings of the 5th International IBPSA conference, 1997, pp. 7-14.

[37] G. Lefebvre, E. Palomo, A. Ait-Yahia, Substructured modeling of linear thermal systems: the model Synthesis, Numer Heat Transf Part B Fundam 39 (2001) 303-324, http://dx.doi.org/10.1080/10407790151075012 ISSN 1040-7790, doi: $\backslash$ bibinfo.

[38] A. Gafsi, G. Lefebvre, A new inverse method using model Synthesis: application to thermal systems, Numer Heat Transf Part B Fundam 49 (6) (2006) 513-536, http:// dx.doi.org/10.1080/10407790500344001 ISSN 1040-7790, doi:\bibinfo http:// www.tandfonline.com/doi/abs/10.1080/10407790500344001.

[39] S. Tada, Material design of aerated concrete - an optimum performance design, Mater Struct 19 (1) (1986) 21-26.

[40] StarWest, EDStaR framework, (2016) http://edstar.lmd.jussieu.fr/.

[41] J. Delatorre, G. Baud, J. J. Bézian, S. Blanco, C. Caliot, J. F. Cornet, C. Coustet, J. Dauchet, M. El Hafi, V. Eymet, R. Fournier, J. Gautrais, O. Gourmel, D. Joseph, N. Meilhac, A. Pajot, M. Paulin, P. Perez, B. Piaud, M. Roger, J. Rolland, F. Veynandt, S. Weitz, Monte Carlo advances and concentrated solar applications, Sol. Energy ISSN 0038092X, doi: \bibinfo\{doi\} \{https://10.1016/j.solener.2013.02.035\}, URL http://linkinghub.elsevier.com/retrieve/pii/S0038092X13001448.

[42] C. Spiesser, Suitable method for complex systems simulation: self-adaptive and selflearning proof-of-concept applied to coupled heat transfer, Ph.d. thesis IMT - Ecole Nationale Supérieure des Mines d'Albi-Carmaux, 2017. 